\title{
El acceso racional a Dios en la Institución de la Religión Cristiana de Juan Calvino
}

\author{
MANFRED SVENSSON \\ Universidad de los Andes (Chile) \\ msvensson@uandes.cl
}

\begin{abstract}
Resumen
El presente artículo analiza los primeros cinco capítulos de Institución de la Religión Cristiana, discutiendo algunas de las principales interpretaciones que se ha ofrecido de la doctrina del sensus divinitatis ahí presentada por Calvino, y preguntando por su general pertenencia a una tradición de fe en búsqueda de comprensión.
\end{abstract}

Palabras claves: Calvino, teología natural, sensus divinitatis.

\section{Rational access to God in John Calvin's «Institutes of the Christian Religion»}

\begin{abstract}
The present article presents an analysis of the first five chapters of John Calvin's Institutes of the Christian Religion, and discusses some of the main interpretations that have been advanced concerning the doctrine of the sensus divinitatis that Calvin espouses in this work.
\end{abstract}

Key words: Calvin, natural theology, sensus divinitatis.

Doctor en Filosofía por la Ludwig-Maximilians-Universität, Munich (Alemania). Entre sus publicaciones cabe mencionar los libros Theorie und Praxis bei Augustin (2009), Resistencia y gracia cara: el pensamiento de Dietrich Bonhoeffer (2011) y Más allá de la sensatez. (2011), así como los artículos “Adiaphora en san Agustín?” (2010), “A Defensible Conception of Tolerance in Aquinas?" (2011) y "Conciencia moral y libertad de conciencia en Locke" (2011). 


\section{Introducción}

El presente artículo busca presentar la posición de Calvino respecto del acceso racional a Dios por vías distintas de la revelación especial. Esto es, si bien el acceso a Dios por dicha vía de revelación especial también podría ser descrito como un «acceso racional», aquí nos limitamos a la pregunta por la pertenencia o eventual distancia de Calvino respecto de una ampliamente concebida tradición de teología natural. Por lo mismo, nuestra aproximación, si bien busca primordialmente ser una interpretación de los pasajes relevantes de la obra de Calvino, al mismo tiempo intentará presentarlo dentro del marco más amplio de dicha tradición y su pertenencia a ella.

La pregunta así abordada tiene por supuesto un interés tanto sistemático como histórico. Es verdad, naturalmente, que lo dicho por Calvino, Lutero o Melanchthon sobre la posibilidad de demostrar la existencia de Dios no nos dice nada sobre la legitimidad de los argumentos mismos que hoy se usa para defender o rechazar su existencia. Sin embargo, las posiciones defendidas por los reformadores - $\mathrm{O}$ interpretaciones contemporáneas de las mismas- se encuentran constantemente presentes en la discusión actual, en algunos casos bloqueando, en otros casos estimulando la reflexión filosófica cristiana. Esto no debiera extrañar a nadie: los hombres tenemos un fuerte apego a nuestras tradiciones, y el eventual irracionalismo de los reformadores puede ser un significativo motivo para que cristianos de tradición protestante tengan dudas respecto de cómo emprender su propio trabajo en la filosofía, o bien para que la vean estimulada en caso de no existir tal irracionalismo. Para quienes se encuentran en tal posición, la aclaración histórica desempeña un papel más que histórico. En el mismo sentido, una clarificación de este trasfondo parece ser potencialmente iluminadora respecto de cuánto pueden hacer en común hoy filósofos de distintas tradiciones cristianas: tal trabajo en común podría revelarse como algo que en realidad no obedece de modo exclusivo a un ímpetu ecuménico contemporáneo, sino que obedece a la existencia de una tradición intelectual cristiana en común que, con todas las transformaciones que pueda haber padecido por el quiebre eclesiástico del siglo XVI, subsiste como un robusto tronco supraconfesional.

Junto a ese tipo de auxilio prestado al desenvolvimiento de la filosofía por parte de los cristianos en el escenario contemporáneo, una adecuada comprensión del sensus divinitatis calviniano también puede contribuir a nuestra evaluación de la historia de la filosofía. Así ocurre, por ejemplo, en relación a diversas posiciones en la filosofía contemporánea que buscan apoyo en este tipo de antecedentes 
históricos. Las ideas de Platinga (1998: 296-315) respecto de la naturaleza del trabajo filosófico de los cristianos, por ejemplo, guardan estrecha relación con su propia reconstrucción del sensus divinitatis calviniano, y ésa es una pretensión cuya evaluación por supuesto presupone una adecuada revisión de tal eventual precedente. Pero más allá de esta preocupación por el vínculo de Calvino con posiciones contemporáneas, se suele asignar a la Reforma protestante un papel importante en el desarrollo intelectual de Occidente, y la discusión respecto de la pertenencia de Calvino a la tradición precedente en lo que se refiere al acceso racional a Dios sin duda puede arrojar significativa luz sobre el pretendido ímpetu dado por la Reforma al pensamiento moderno.

Respecto de dicho último punto existe una imagen convencional de una fuerza abrumadora. Según ésta, los reformadores habrían acentuado la distancia entre Dios y el hombre, o la incapacidad de conocerle por parte del hombre caído, de modo tal que el abismo abierto entre el hombre y Dios sólo podría ser cruzado por la fe (Haakonsen, 1996: 25). Así, según la perspectiva desde la que se escriba, se suele otorgar a los reformadores un sitial de honor o de deshonor, pero en cualquier caso un lugar significativo en la ruta que ha llevado a desesperar del acceso racional a Dios. Esto suena plausible, por supuesto, pues si la condición caída del hombre y la justificación por la fe son énfasis típicamente protestantes, parece haber doble motivo $-\mathrm{y}$ por aspectos centrales para la propia confesión de fe-por los que el acceso racional a Dios podría quedar imposibilitado. Pero ya antes de entrar en el análisis detallado, cabe hacer algunas precisiones respecto de este tipo de obstáculos generales. En primer lugar, cabe notar que una expresión como «depravación total» sólo llega a ser usada en tiempos muy recientes para describir la comprensión calvinista del hombre caído. Los textos del siglo XVI y XVII, en tanto, ciertamente contienen terminología que busca volver patente la condición humana, pero la «totalidad» de la corrupción designa ahí claramente la extensión, no la intensidad de la misma. Por decirlo de otro modo, lo que está en cuestión no es que seamos todo lo que malos que cabe ser, sino que la maldad se ha extendido a cada parte de nosotros. Y tal modo de hablar al respecto implica que si bien habrá consecuencias epistémicas del pecado, no hay motivo para traducirlas en una imposibilidad del acceso racional a Dios. De modo análogo cabe precisar el sentido de la doctrina de la justificación por la fe: que sea mediante la fe que somos justificados no dice nada respecto de cómo conocemos a Dios. Si el solafideísmo fuera una tesis respecto de nuestra relación cognitiva con el Creador, y no aquello por lo que los creyentes vuelven suyo el triunfo de Cristo en la cruz, sería justificada la presunción de que todo acceso racional a Dios está cerrado. Pero a tal 
interpretación se opone el sencillo hecho de que estamos ante una doctrina de la justificación.

Cabe, pues, una renovada mirada a las correspondientes páginas de Juan Calvino, una mirada liberada del prejuicio y vuelta más precisa por el avance tanto en la investigación histórica sobre Calvino (Muller, 2000) como en la evaluación filosófica de su obra (Helm, 2004). Partiremos por considerar las declaraciones de intenciones de Calvino en relación al conocimiento de sí y de Dios, que encabezan las sucesivas ediciones de Institución. Luego dirigiremos la mirada a la concepción calviniana del sensus divinitatis, para en una última sección considerar el tipo de conocimiento alcanzado, en particular en relación a la pregunta por los elementos teóricos y prácticos del mismo.

\section{Conocimiento de sí y de Dios en la Institución}

Nuestra investigación estará centrada en las primeras páginas de Institución, primordialmente en la edición definitiva de 1559. Es notable que una prestigiosa Historia de los Dogmas dentro de la tradición católicoromana reconozca en estas páginas "pasajes que casi suscitan la impresión de un tratado de filosofía de la religión o de historia de la religión» y manifieste además reconocimiento a «las apelaciones a los Padres de la Iglesia o incluso a los escolásticos» (Beumer, 1977: 95). Los elementos patrísticos en la obra de Calvino y de los reformadores en general no constituyen para nadie una sorpresa (Lane, 1999), pero el reconocimiento de elementos escolásticos puede parecer sorprendente. Con todo, cabe señalar que en eso la citada historia de los dogmas coincide con los mejores resultados de la investigación contemporánea sobre Calvino. Como en todos los reformadores, abundan por supuesto en Calvino las observaciones críticas sobre los escolásticos. Pero el fino análisis histórico de Richard $\mathrm{A}$. Muller ha mostrado que con tal término Calvino casi siempre hace referencia a contemporáneos suyos, y no a escolásticos de los XIII o XIV. En efecto, en las versiones francesas que el propio Calvino hizo de su obra, tales pasajes son rendidos como referencias a théologiens Sorboniques o Sorbonnistes (Muller, 2000: 50-51). De modo paralelo, Paul Helm ha llamado la atención sobre los considerables paralelos sistemáticos con clásicas posiciones escolásticas, al margen de cualquier efectiva conexión histórica (Helm, 2004: 11-34). Así ocurre de modo regular con distinciones como aquélla entre el conocimiento de Dios en sí y quoad nos. Pero a esto podemos añadir que hay otro sentido muy significativo en que Calvino se encuentra dentro de una tradición escolástica, a saber, el hecho de que se trata de una producción 
primordialmente pedagógica, de formación de escuela. De modo similar al proemio de Tomás de Aquino a la Summa Theologiae, donde éste declara que la intención de la obra es incipientes erudire, Calvino afirma en el prefacio la Institución de 1536 que «sólo buscaba presentar ciertos rudimentos que permitan formar en la piedad a los que son tocados por alguna búsqueda de religión» (praef., 9) ${ }^{1}$. Se trata de una obra pedagógica $y$, tal como la Summa Theologiae - pero no la Summa contra Gentiles-, dirigida a los que ya se encuentran dentro del camino de la fe.

Pero a pesar de esa intención que Tomás de Aquino y Calvino tienen en común, hay aquí una significativa diferencia, dada por el contexto polémico de la obra de Calvino: el mismo prólogo de Institución testifica de que ya en dicho temprano momento de la Reforma, los protestantes eran criticados por estar subvirtiendo no sólo las obras meritorias y diversos puntos doctrinales en discusión, sino también la luz natural de la razón. Se trata de una acusación que todavía hoy se encuentra con frecuencia entre destacados pensadores católicos (Maritain, 1986: 40-45; Finnis, 2011: 166) y que, según aquí se puede constatar, no es fruto de los estereotipos creados durante la época de las guerras de religión y la confesionalización, sino que data de los inicios mismos de la Reforma. Calvino nombra, en efecto, dicha acusación, y de un modo que parece conceder cierta razón a los acusadores, pues al nombrar la objeción se refiere a esta luz como «no sé qué luz ciega de la naturaleza» (praef., 13). Una parte significativa del problema que aquí abordamos pende de cómo uno se disponga a abordar afirmaciones como ésa. La literatura del siglo XVI ciertamente es de un tono distinto que la del siglo XIII, y no siempre es fácil saber en qué medida tomar una palabra como un término técnico, o cuán omnicomprehensivas considerar ciertas críticas. En ocasiones el mismo Calvino nos permite notar esta dificultad, y la noción de libre albedrío puede servir de elocuente ilustración. Tanto el término como el concepto de libre albedrío suelen ser tenidos por ajenos a la obra de Calvino, como cosas que incluso por definición tendrían que quedar fuera de su obra, y por los mismos motivos que la luz natural de la razón. Pero cuando Calvino manifiesta su expresa decisión de no usar el término libre albedrío, concede sin embargo que otros lo sigan usando siempre que con ello no se transmita una concepción pelagiana. En tal caso, afirma «no serán molestados» por él, aunque ciertamente considera

1 Las referencias a Institución (Inst.) remiten en el presente artículo al texto editado por Peter Barth et al. Johannis Calvini Opera Selecta. Referencias a otras obras de Calvino remiten a la edición de Wilhelm Baum et al., contenidas en el Corpus Reformatorum (59 vols. de Calvini Opera, abreviado CO). Lutero es citado conforme a la edición de Weimar (abreviado WA) y Melanchthon siguiendo la Studienausgabe (abreviado MWA). 
más sabio derechamente acabar con el uso del término (Inst. II, 2, 8; sobre dicho problema véase el análisis de Lane, 1981: 72-90). Hay pues un sentido, y uno nada de trivial, en el que cabe decir que Calvino cree en la existencia del libre albedrío, a pesar de su reticencia ante este término. Así lo entiende también el grueso de la tradición calvinista que, manteniendo la precaución ante el pelagianismo, afirma tanto en los textos confesionales como en la obra de sus principales representantes la existencia de la libertad humana (Confesión de Fe de Westminster, cap. 9, y van Asselt, 2010). Algo similar parece poder decirse de su impresión respecto de la capacidad racional del hombre en 1536. La ceguera que aquí Calvino atribuye a la luz de la naturaleza puede, en efecto, ser restringida a un área: puede estar simplemente hablando de la ceguera de la razón natural en relación a la salvación, lo cual es distinto de declararla ciega en relación al conocimiento de Dios como Creador. Ésta parece ser la explicación más razonable, pues la doctrina del sensus divinitatis, que a continuación expondremos, se encuentra presente desde las tempranas ediciones de Institución.

En cualquier caso, ese tipo de afirmaciones sobre la ceguera de la razón desaparecen del todo de los prólogos subsiguientes: en adelante la expresión «luz natural de la razón» no le causará problemas a Calvino; si respecto del libre albedrío se puede decir que abraza el concepto pero no el término, en el caso de la razón natural luso el término deja de ser problemático. En efecto, la breve declaración del argumento de la obra, añadida a la edición francesa que en 1545 hiciera de la versión latina de 1543, sigue manifestando como su propósito el auxiliar a los principiantes (subvenir aux simples), pero Calvino afirma ahora más claramente que eso se hará presentando en orden los principales tópicos de la «Philosophie Chretienne» (Argument du present livre, 7), expresión que parece situarlo más cerca del centro de la tradición y más claramente alejado de cualquier posición fideísta o irracionalista. Tal expresión puede parecer demasiado poco indicio para hablar de una evolución; pero la carta al lector del mismo año termina con una cita de la carta 143 de Agustín que invita a pensarlo en esos términos: «Yo soy de los que escriben progresando y progresan escribiendo». Dirijámonos pues a la edición final de 1559.

Esta edición comienza, como todas las anteriores, con la afirmación de que la suma de la sabiduría se encuentra en un doble conocimiento, el de Dios y de nosotros mismos (Inst. I, 1, 1. Dei cognitione et nostri). Esto puede evocar en nosotros el dictum de los Soliloquios agustinianos, según el cual Agustín solo buscaba conocer a Dios y al alma (sol. I, 2, 7). Pero este paralelo debe ser trazado con cuidado, sobre todo respecto del segundo punto, el conocimiento del alma. Pues Calvino no está pensando en un 
conocimiento de su naturaleza, sino de su condición actual: la suma de la sabiduría consiste en saber sobre nuestra miseria (pravitas, corruptio), para así dirigirnos al Creador (Inst. I, 1, 1). Éste es pues el punto en que con toda naturalidad debería aparecer la negación de una luz natural del intelecto humano, y la consiguiente necesidad de acceder a Dios por otra vía.

Ahora bien, efectivamente aquí uno encuentra términos como pravitas o corruptio para designar el estado del hombre. Pero conviene detenerse y preguntar en qué medida son términos técnicos que estén designando una efectiva corrupción de la naturaleza en estrictísimo sentido -tal que la hiciera incapaz de ejecutar cualquiera de sus funciones- y en qué medida se trata más bien de términos que simplemente quieren volver muy patente la caída. Me parece claro que esto último es lo correcto. También en Institución II, 2, el capítulo en que más detenidamente discute la miseria actual del hombre (naturalium donorum corruptio, pravitas voluntatis, etc.), afirma que la razón, "siendo un don natural, no pudo ser borrada del todo» (Inst. II, 2, 12). Hay según Calvino un sensus propriae pravitatis, no una mera pravitas, y ese sentido de la propia miseria, escribe Calvino, nos llevará a «reconocer que sólo en el Señor está la verdadera luz de la sabiduría» (Inst. I, 1, 1). Lo menos que tiene que estar conservado en el hombre es pues tal sensus.

Pero eso no significa que estemos ante un conocimiento de Dios de carácter autónomo. Calvino más bien pasa de inmediato a llamar la atención sobre el recíproco condicionamiento del conocimiento de Dios y el conocimiento de sí mismo. Ha partido por indicar que la conciencia de nuestra miseria lleva a un mayor conocimiento de Dios, pero ahora indica que ese reconocimiento de la propia miseria presupone desde luego una medida suficientemente elevada a la luz de la cual mirarse: se requiere primero haber dirigido la mirada al rostro de Dios y desde ahí volver a descender a la consideración de nosotros mismos (Inst. I, 1, 2). $\mathrm{Al}$ conocimiento de Dios no se llega pues desde la mera comprensión de la propia miseria, sino que de algún modo el conocimiento de Dios está ya en nosotros haciendo así posible el reconocimiento de que nuestra condición es miserable. ¿Cómo concibe Calvino dicho conocimiento? Para abordar dicha pregunta nos centraremos a continuación en los capítulos 3 y 5 del primer libro de Institución. El primero de éstos está dedicado al sensus divinitatis que según Calvino todos los hombres poseen, el segundo al conocimiento de Dios a partir de sus obras. 


\section{El acceso a Dios: sensus divinitatis y conocimiento desde la creación}

La posibilidad de un acceso racional a Dios que no sea por la vía de la revelación especial se topa no sólo con las objeciones que hasta aquí hemos mencionado, centradas en la idea de que una depravación total del hombre como sería profesada por la tradición calvinista. También hay objeciones propias del tipo de «cristocentrismo» propio de la escuela barthiana, que proyecta hacia Calvino sus propias tesis respecto de la imposibilidad de un conocimiento de Dios que no sea a través de Cristo (Busch, 2008: 222). Ahora bien, parece haber algún sustento textual en Calvino para tales tesis. Así, por ejemplo, tenemos la afirmación según la cual respecto de las «cosas celestiales» los hombres son ciegos (Inst. II, 2, 13 y 18), que desde luego recuerda lo que hemos visto en el prólogo de 1536 sobre la ceguera de la razón natural. La imprecisión de la expresión «cosas celestiales» podría inducirnos a pensar que Calvino nos declara ciegos respecto de todo conocimiento de Dios; pero significativamente, la afirmación se encuentra en el libro II de la obra, expresamente dedicado al conocimiento de Dios en cuanto redentor. Las «cosas celestiales» no son las que podemos saber sobre la creación de Dios, sino sobre el reino de Dios (Inst. II, 2, 18). Este género de afirmación no parece pues de modo alguno minar el conocimiento que se pueda tener sobre el Creador.

En gran medida, la cuestión parece poder resolverse mediante aclaraciones conceptuales, aclaraciones que por lo demás no descansan sobre tesis muy sutiles, sino en estructuras básicas de la teología, como la relación con Dios como Creador y como Redentor, distinción que en este caso incluso da la estructura a la primera mitad de la Institución de Calvino. El hecho de que «todo conocimiento de Dios al margen de Cristo sea un abismo inmenso que absorbe todo nuestro sentido» (CO 55: 226), afirmación que Busch cita a su favor, no parece contrariar lo precedente. Eso permite, más bien, dejar firmemente establecida la tesis contraria: Calvino no está negando la posibilidad de tal conocimiento, sino precisamente describiendo en qué condición se encuentra el hombre que accede de tal modo a Dios - una condición de desesperación, por verse enfrentado a algo que «absorbe todo su sentido»-, con lo cual precisamente se da por sentado que tal conocimiento es en realidad posible. Se trata, por lo demás, de una posición que se encuentra también en Lutero. En efecto, aunque en la obra del reformador alemán esto coexista con una considerable cantidad de afirmaciones en sentido aparentemente contrario, que hacen más ardua su interpretación, tampoco en él es poco común encontrarse con la afirmación de que «el 
conocimiento de Dios es doble, de tipo general o específico, y el general lo tienen todos los hombres: que es Creador del cielo y de la tierra, que es justo, que castiga a los impíos, etc.» (WA 40, 1: 607).

Puede tal vez considerarse que hay cierto cambio de énfasis aquí entre los reformadores y la tradición precedente. Que lo alcanzado por la razón natural es un conocimiento cierto, pero ínfimo y no salvífico, es algo que unos y otros confiesan a una voz. Como lo expone Calvino en uno de sus comentarios bíblicos, «la naturaleza de tal testimonio [de la creación a favor de la existencia de Dios] es tal, que priva a los hombres de excusa, pero no basta para salvación» (CO 48: 327). Pero por el lado de los reformadores, podemos ver que el tono usual es el de recalcar que este conocimiento no sólo no es beatífico, sino que derechamente conduce a desesperación. No conviene minimizar dicho énfasis; con todo, es un énfasis, y uno tal que no deja lugar a dudas respecto de la posibilidad de conocer al Creador. Es más, está en cuestión no sólo la posibilidad, sino de la necesidad de tal conocimiento, ya que lo producido por él es una desesperación a la que los reformadores creen que debemos encaminarnos, para desde ahí buscar el conocimiento verdaderamente salvífico.

Podemos, pues, dejar de lado dicho tipo de objeción, y atenernos a lo que palmariamente encontramos al comienzo de Institución: la convicción de Calvino de que hay en todos los hombres un sensus divinitatis que -añade enfáticamente en la misma línea que por primera vez menciona este sensus - «no puede ser borrado» (Inst. I, 3, 1). La expresión sensus divinitatis, sensus deitatis o semen religionis se encuentra en varios lugares de la edición de 1559. Aquí, en el tercer capítulo, parte por decir que este sensus divinitatis o naturalis instictus es algo indubitable, extra controversiam. Esto puede parecer una afirmación osada o al menos superada, pues desde los tiempos de Calvino se ha vuelto cada vez más controversial la creencia en la existencia de Dios, y tanto más la creencia en que sea una posición racionalmente defendible. Conviene, por tanto, ver los pasos que Calvino sigue para afirmarlo. Creo que se puede distinguir tres pasos. En primer lugar, propone lo que podríamos considerar un sencillo axioma teológico: Dios no puede haber dado lugar a que nos refugiemos en la pretensión de ignorancia respecto de Él, y por eso ha puesto este sensus divinitatis en nosotros. En segundo lugar, encontramos lo que parece ser una observación empírica: la convicción de que de hecho el teísmo - en cualesquiera aberrantes variantes- se encuentra universalmente extendido. Para esto Calvino invoca testimonios paganos, particularmente de Cicerón (Ethnicus ille), en que se busca mostrar que la religión es algo que se extiende incluso a los pueblos que más se asemejan en lo restante a las bestias (Inst. I, 3, 1). 
Esto indica claramente, por si cabe alguna duda, que Calvino está hablando de un conocimiento existente también en la condición caída del hombre. ¿Pero tiene razón? Este segundo punto es probablemente el más débil, pues una sola instancia de ateísmo parecería bastar para desmentirlo. Es un argumento empírico, y abierto por tanto a refutación empírica. El hecho de que Calvino no parezca verlo en modo alguno como un mal argumento, o como eventualmente refutable, se relaciona con seguridad con el tercer punto: Calvino sostiene aquí que la idolatría es un indicio claro de la existencia de este sentido de la divinidad. Que el hombre, a pesar de su resistencia a considerar otras criaturas como superiores a él mismo, les rinda culto, es para Calvino un indicio "sumamente vehemente" de lo imposible de obliterar que es esta impresión de la divinidad. En su clásica afirmación, el corazón humano es una fábrica de ídolos, y Calvino toma esto como un claro indicio de la existencia de este sensus. En un capítulo posterior responde de modo similar a los que - en una suerte de temprana filosofía de la sospechainterpretarían la religión como fruto de una conspiración sacerdotal. Tal conspiración puede de hecho existir, concede Calvino, pero «jamás lograría su propósito si las mentes de los hombres no estuvieran antes imbuidas de aquella constante persuasión respecto de Dios, de la cual como de semilla emerge la propensión a la religión» (Inst. I, 3, 2). A estos tres argumentos presentados inicialmente por Calvino, se añaden luego otros indicios como el temor de Dios y los terrores de la conciencia provocados por nuestro actuar - una suerte de argumento moral a favor de la existencia de Dios (Inst. I, 3, 2 y 3).

Pero aquí se nos plantea un problema de alguna dificultad: pues algunos de los puntos anteriores sugieren que lo que tenemos por este sensus es conocimiento de Dios - así ocurre, por ejemplo, en esta última referencia a la conciencia-, mientras que otros puntos parecen más bien sugerir que tenemos la capacidad de adquirir el conocimiento, pero que de hecho no lo obtenemos. Esta distinción no siempre es precisada, y así no es poco común encontrar en la literatura secundaria referencias a este sensus divinitatis como una especie de conocimiento innato de Dios. No parece fácil solucionar esto: se podría argumentar que Calvino usa precisamente la expresión sensus, y que si bien no hay por qué imaginar esto literalmente como un sentido, en analogía a los cinco sentidos externos, podríamos entenderlo como una disposición al conocimiento de Dios. Pero sensus también podría significar no el sentido en cuanto facultad o disposición, sino precisamente como lo sentido; como hemos visto, Calvino usa de modo equivalente expresiones como «semilla»o «persuasión». En dicho caso, parecería tratarse de un conocimiento. 
Esto es hoy disputado no tanto por parte de críticos católicos, sino por parte de quienes en el calvinismo contemporáneo buscan distanciarse de lo que diagnostican como el carácter intelectualista de la modernidad. Así, en medio del intento por desvincular la propia tradición de tal tendencia, se afirma que el sensus divinitatis no está concebido por Calvino como «una disposición intelectual a formar creencias teístas, sino como una apasionada tendencia a adorar» (Smith, 2009: 122-123). Pero este resguardo, obedeciendo a una agenda contemporánea, fácilmente puede desequilibrar nuestra lectura de Calvino. Pues él es tan consciente como la tradición previa del requisito de cierto conocimiento para que pueda haber amor, y lo mismo puede decirse de la adoración. Ahora bien, no es menos cierto que en ocasiones Calvino habla como si sólo fuese genuino conocimiento uno que desemboca en adoración. Sobre este aspecto de la discusión tendremos que volver más adelante.

Ahora bien, sea que entendamos el sensus como una disposición o como un conocimiento efectivamente poseído, la inexcusabilidad de los hombres queda resguardada, pues Calvino afirma que Dios está activo, continuamente renovando la memoria de este conocimiento y añadiendo nuevas gotas del mismo (Inst. I, 3, 1). Esta referencia al actuar de Dios desde fuera de nosotros para impartir tal conocimiento nos conduce al capítulo quinto de la obra, donde tenemos a Calvino tratando respecto de cómo el conocimiento de Dios se nos presenta en la creación y en su gobierno del mundo. La popular traducción inglesa de Beveridge habla aquí de las pruebas (proofs) que tendríamos tanto en la sencilla contemplación del mundo como en el conocimiento más preciso que nos dan la medicina, la astronomía y la física. También la clásica traducción española realizada por Cipriano de Valera en 1597 habla aquí de «infinitas pruebas». Pero ésa no es la terminología exacta de Calvino, quien habla no de pruebas, sino de «testimonios» (Inst. I, 5, 2, documenta... quae testentur... esse testes). A partir de tales testimonios se puede, tal vez, elaborar pruebas a posteriori, pero ellos mismos no constituyen tales pruebas. Además, otro pasaje del mismo capítulo sí incluye la palabra demonstratio, pero precisamente para descartar que sea necesaria: «Vemos que no hay necesidad de una larga y difícil demostración para mostrar los testimonios que sirven para ilustrar y afirmar la majestad divina» (Inst. I, $5,9)$. Conviene detenernos a discutir este punto con algún detalle, pues muchos consideran imposible que desde una perspectiva calvinista se favorezca la argumentación a favor de la existencia de Dios. ¿Es tal conclusión lo que avalan textos como éste?

Hay una cosa que puede concluirse con cierta facilidad: ni aquí ni en general en la obra de Calvino nos encontramos con tales pruebas. Pero 
tampoco nos encontramos con un rechazo de las mismas. O, más bien, no nos encontramos con un rechazo de la posibilidad de las mismas, sino con un rechazo de su necesidad. Ante eso cabe hacer dos preguntas: una respecto de por qué no serían necesarias, otra respecto de si acaso serían posibles. Respecto del primer punto parece claro el móvil de Calvino: está hablando de un conocimiento que todos poseemos, y en particular con miras a nuestra inexcusabilidad. Esto se vería por supuesto comprometido si nos viéramos obligados, para conocer a Dios, a pasar por las dificultades de una prueba racional. Que éste es el motivo para no considerar necesarias las pruebas puede ser confirmado por otra vía. Como ha hecho notar Michael Sudduth (1995: 53-67), en su comentario a Hechos de los Apóstoles, Calvino escribe que Pablo y Bernabé no ofrecieron «un discurso de tipo sutil y filosófico respecto de los secretos de la naturaleza, pues su discurso se realizaba ante el pueblo inculto» (CO 48: 328). Se trata pues de un motivo contingente por el cual no desarrollaron dichas pruebas. Pero es un motivo contingente que se repite en la audiencia de Calvino, pues como hemos visto escribe su obra para principiantes. Por lo demás, en un pasaje cercano, que Sudduth omite, Calvino añade que «sabemos que el orden del aprendizaje requiere partir desde las cosas que resultan más conocidas para nosotros» (CO 48: 326). No sólo indica, pues, un motivo contingente para explicar la ausencia de pruebas en el caso de Pablo y Bernabé (y por extensión en su propio caso), sino que esto ocurre en un contexto que, aunque se trate de un comentario bíblico, muestra cierta autoconciencia metodológica.

La validez de esta interpretación puede verse reafirmada si planteamos la pregunta por la posibilidad de tal argumentación. Lo primero que cabe afirmar al respecto, es que no se encuentra en Calvino una sola afirmación que permita sospechar que considere imposible desarrollar argumentos a favor del teísmo. Creo, además, que se puede indicar al menos dos indicios que los volverían aceptables. En primer lugar, debe hacerse mención de la atención que él presta al tipo de discurso requerido por distintas audiencias. Pues en el capítulo cinco, Calvino hace mención, por única vez en estos capítulos iniciales, de la existencia de un público más sofisticado, pero precisamente como un hecho del que aquí no se hará cargo: puede haber un conocimiento más profundo de los misterios de Dios en quienes han sido introducidos en las artes liberales, escribe, pero nadie, aunque las ignore, está desprovisto al menos de aquel conocimiento de Dios que debiera ser suficiente para movernos a la adoración del mismo (Inst. I, 5, 2.). Esto parece implicar que entre quienes se dedican a dichas disciplinas es posible un conocimiento de un tipo distinto, basado en inferencias, partiendo de los mismos testimonios externos que el resto. En segundo lugar, se puede 
mencionar que no es extraño encontrar en Calvino inferencias respecto de las perfecciones de Dios, afirmaciones como que «de su poder somos llevados (deducit) a pensar en Su eternidad, porque es necesario que Aquel del que todo deriva su origen sea eterno y principio de sí mismo» (Inst. I, $5,6)$. No puedo ver motivo por el que alguien que acepta este tipo de raciocinios respecto de la naturaleza de Dios habría de considerarlos por principio inaceptables respecto de la existencia del mismo. La posibilidad de un acceso argumentativo a Dios se mantiene así abierta, y es algo que se podría desarrollar en perfecta continuidad con Calvino, aunque él mismo no se haya dedicado a tal tipo de proyecto.

\section{El conocimiento alcanzado}

Visto esto, podemos intentar aclarar de qué tipo es el conocimiento alcanzado por esta vía. Sobre eso Calvino ya dice algo antes de mencionar la existencia del sensus divinitatis. En efecto, el capítulo segundo de la obra está dedicado a afirmar la existencia de una duplex cognitio de Dios, la diferencia entre conocerlo como Creador y como Redentor. El texto inequívocamente afirma que como redentor nuestra razón no alcanza a conocerlo. Pero es igualmente evidente que sí es presentado como cognoscible en cuanto Creador (Inst. I, 2, 1). Esto no quita que también para el adecuado conocimiento de Dios como Creador sea necesario recurrir a la Biblia (Inst. I, 6, 1). Pero cabe preguntarse si acaso Calvino está pensando solo en una diferencia de grados, o si cree que las Escrituras añadirían algo cualitativamente distinto al conocimiento de Dios como Creador. Esto no parece de fácil respuesta, pero en el capítulo 10 de Institución, tras mencionar que los atributos divinos se encuentran cuidadosamente presentados en el salmo 145, Calvino afirma que «ahí no hay nada que no pueda ser encontrado en las criaturas» (Inst. I, 10, 2). Lo implicado parece ser que si bien mediante la revelación se conoce con mayor profundidad los atributos divinos, la creación ya nos pone ante la totalidad de ellos. Ahora bien, esto va de la mano no sólo de la afirmación de lo limitado que es tal conocimiento, sino también de la conciencia de que «Su esencia es incomprehensible, escapando su numen a todo sentido humano, aunque la gloria brille en cada una de sus obras» (Inst. I, 5, 1). En esta afirmación de que la esencia de Dios se escapa al conocimiento humano, una vez más podemos constatar que Calvino se está inscribiendo en una larga tradición que, al menos desde Gregorio Nacianceno, ha distinguido la posibilidad de conocer la existencia divina de la posibilidad de conocer su esencia. 
Pero no sólo podemos preguntar por el tipo y grado del conocimiento alcanzado, sino también respecto del fin por el que se busca el conocimiento de Dios. En este punto se atribuye con frecuencia un énfasis práctico a la Reforma, en contraste con la caracterización de la teología como ciencia teórica por parte de Tomás de Aquino y otros medievales. Así por ejemplo Leo Strauss, en su introducción al estudio de la filosofía medieval, ha llamado la atención sobre el contraste entre el título de la principal obra teológica del siglo XIII y la principal del siglo XVI: por una parte la Summa Theologiae, por otra parte la Institución de la Religión Cristiana - contraste al que ha dado un significado epocal, como representativo de una preocupación por una parte por lo que Dios es en sí mismo, por otra por lo que es para nosotros (Strauss, 2007: 309). Pero ambos polos de tal contraste son problemáticos, tanto más si se piensa en las asociaciones que tal caracterización produce en la mente de personas menos educadas que Strauss. Por una parte, por el hecho de que ciencia teórica no es para Tomás de Aquino una ciencia que no cambie al cognoscente, sino una que puede cambiar al cognoscente sin cambiar el objeto conocido. Por otra parte, porque «énfasis práctico» también puede significar cosas muy diversas. Podría, en este caso, significar cierto énfasis puramente soteriológico en la teología, y tal vez habría algo de justicia al caracterizar de ese modo a Lutero o a parte del luteranismo: «Conocer a Cristo es conocer los beneficios de Cristo», escribe Melanchthon en una célebre afirmación de sus Loci communes (MWA II: 7). En contraste con eso, conviene precisar que si Calvino sólo llama conocimiento de Dios a un conocimiento con un significativo componente práctico, se trata de algo distinto de estas posiciones de Lutero y Melanchthon. En palabras del propio Calvino «no comprendo bajo conocimiento de Dios uno por el que solo entendemos que existe un Dios, sino uno por el que entendemos a la vez qué nos compete saber de él, qué sirve para su gloria» (Inst. I, 2, 1). A tal modo de abordar la cuestión se le puede tal vez calificar de práctico, pero debe enfatizarse que sigue tratándose de algo marcadamente teocéntrico, de un modo que hace perder bastante sentido al contraste establecido por Leo Strauss.

Pero el vínculo del conocimiento de Dios con la adoración permite algunas precisiones más. Es frecuente que Calvino restringa el conocimiento de Dios a uno que lleve a la adoración. Es decir, no sólo afirma que el conocimiento de Dios nos debe llevar a darle gloria, sino que sólo califica de conocimiento propiamente tal al que tenga tal fruto. Resulta llamativo que tampoco esto lo entiende Calvino como un énfasis exclusivo del cristianismo. Por el contrario, en una de las primeras páginas de Institución afirma que ya los filósofos descubrieron que sólo podía llamarse conocimiento de Dios uno que condujera a la adoración; 
y de inmediato nos indica de modo claro a qué se está refiriendo: a la doctrina de la theosis o homoiosis theou. «No otra cosa quiso Platón - escribe Calvino- al frecuentemente enseñar que el sumo bien del alma es la similitud con Dios, cuando por conocimiento de Él, el alma toda es transformada en dirección al mismo» (Inst. I, 3, 3). Algunos capítulos más adelante el mismo Platón es mencionado como el más sobrio y religioso de los filósofos (Inst. I, 5, 11), pero precisamente para indicar que no ha cumplido con ese requisito que él mismo habría puesto, esto es, que incluso en el mejor de ellos, como en todos los hombres, el conocimiento que Dios nos ha dado de sí no da el fruto debido (Inst. I, 5, 11). Esto parece obligarnos a concluir que Calvino habla de conocimiento de Dios en dos sentidos distintos, rara vez precisando cuál usa. Así, en ocasiones califica de conocimiento sólo a aquél que va acompañado de adoración. Cuando habla así, naturalmente afirma a su vez que hemos perdido el conocimiento de Dios. En otras ocasiones, en cambio, como en las primeras páginas de Institución, habla de conocimiento de Dios para calificar algo que permanece a pesar de nuestra falta de adoración. El capítulo cuarto capta bien ambos énfasis, al escribir que «permanece sin embargo aquella semilla que de ningún modo puede ser arrancada de raíz - que hay una divinidad-, pero permanece en un estado hasta tal punto corrompido, que sólo produce los peores frutos» (Inst. I, 4, 4).

\section{Conclusiones}

A la luz de lo que hemos visto es posible sacar una serie de conclusiones. En primer lugar, estamos ante una obra que bien puede entenderse dentro de la gran tradición de una fides quaerens intellectum. Con esto queremos en primer lugar decir que Calvino está inserto no sólo en tal práctica, sino en una tradición de tal práctica. Incluso una mirada superficial a la obra de Calvino basta para convencerse de cuán profundamente está recibiendo no sólo a la tradición patrística, sino de cómo se encuentra presente también un trabajo propio de apropiación del pensamiento antiguo y de los más cercanos escolásticos. Pero eso significa precisamente estar en una tradición viva, moviéndose de un lado a otro dentro de la misma, cultivando él mismo la práctica de reflexionar sobre el estatuto epistémico de las propias creencias.

En segundo lugar, conviene notar que si bien no hay aquí un desarrollo argumentativo tal que permita hablar de modo estricto de una teología natural, no parece que eso se pueda describir como un proyecto incompatible con el de Calvino. Ciertamente lleva la razón Paul Helm al 
notar que el centro de la preocupación de Calvino no es la racionalidad de nuestras creencias sino la inexcusabilidad del hombre (Helm, 1998: 87-107). Pero aunque Calvino está mostrando más que demostrando, es sencillamente falso que su obra ponga obstáculos a un conocimiento demostrativo. Tal precisión es tanto más relevante dada la amplia tendencia a considerar el acceso argumentativo a Dios y la fides quaerens intellectum como caminos alternativos, y no complementarios. Si Anselmo es un representante significativo de la fides quaerens intellectum, tal antítesis ciertamente parece fuera de lugar. También una obra inserta en el camino de una fides quaerens intellectum procede, cuando viene al caso, argumentativamente; y aunque sea una tradición que busca la comprensión mediante un discurso dirigido primordialmente a los creyentes, «este camino para aproximarse a Dios es común a los que están dentro y fuera de la iglesia» (Inst. I, 5, 6).

Por último, cabe señalar que esto arroja significativa luz sobre el vínculo de Calvino con algunos desarrollos posteriores. Algunas décadas atrás, era común la perspectiva que acentuaba la distancia, tanto metodológicamente como en relación a los contenidos, entre Calvino y la escolástica calvinista que se desarrolló hasta fines del siglo XVII. Si bien sigue siendo común encontrarse con el eco de tal perspectiva, hoy se encuentra abundantemente refutada (Trueman \& Clark, 1999; Muller, 2003; van Asselt, 2011). La lectura que aquí hemos presentado de las primeras páginas de Institución se suma en ese sentido a una larga lista de indicios a favor de una tesis más continuista respecto del papel de la Reforma en la historia intelectual de Occidente, esto es, de la continuidad de los reformadores tanto con la escolástica medieval precedente como con la escolástica protestante subsiguiente. Naturalmente, caracterizaciones así de gruesas siguen requiriendo importantes precisiones. Pero también una caracterización así de general servirá como correctivo a una amplia literatura en sentido contrario.

\section{REFERENCIAS}

-Aquino, T. de (1888-1906) Summa Theologiae. En Opera omnia (ed. Leonina, Vols. 4-12). Ciudad del Vaticano: Tipografía Políglota Vaticana.

-Beumer, J. (1977). El método teológico. En M. Schmaus, A. Grillmeier \& L. Scheffczyk (Eds.), Historia de los Dogmas (Vol. I, $\mathrm{n}^{\circ}$ 6). Madrid: BAC.

-Busch, E. (2008). Gott und Mensch. En H. Selderhuis (Ed.), Calvin Handbuch (págs. 222-231). Tübingen: Mohr Siebeck.

-Calvin, J. (1959). Institutes of the Christian Religion (H. Beveridge, Trad.). Grand Rapids: Eerdmans. 
-Calvini, Ioannis (1863-1900). Opera quae Supersunt Omnia [CO] (W. Baum, E. Cunitz \& E. Reuss, eds., 59 Vols.). Braunschweig.

-Calvini, Johannis (1926-1952). Opera Selecta. (P. Barth et al., eds., 5 Vols.). Múnich: Chr. Kaiser.

-Calvino, J. (1952). Institución de la Religión Cristiana (Facsímil de la trad. de Cipriano de Valera, 1597). Buenos Aires: La Aurora.

-Finnis, J. (2011). Religion and Public Reasons. Oxford: Oxford University Press.

-Haakonsen, K. (1996). Natural law and moral philosophy from Grotius to the Scottish Enlightenment. Cambridge: Cambridge University Press.

-Helm, P. (1998). John Calvin, the sensus divinitatis, and the noetic effects of sin. International Journal for Philosophy of Religion (43), 87-107.

-Helm, P. (2004). John Calvin's Ideas. Oxford: Oxford University Press.

-Lane, A. (1981). Did Calvin Believe in Freewill? Vox Evangelica (12), 72-90.

-Lane, A. (1999). John Calvin. Student of the Church Fathers. Edinburgh: T \& T Clark.

-Luther, M. (1883-). Werke. Weimarer Ausgabe [WA]. Weimar: Böhlau.

-Maritain, J. (1986). Tres reformadores: Lutero, Descartes, Rousseau. Buenos Aires: Club de Lectores.

-Melanchthon, Ph. (1951-1975). Melanchthons Werke in Auswabl [MWA]. (R. Stupperich, ed.). Stuttgart-Bad Cannstatt: Gütersloher Verlagshaus Gerd Mohn.

-Muller, R. (2000). The Unaccomodated Calvin. Studies in the Foundation of a Theological Tradition. Oxford: Oxford University Press.

-Muller, R. (2003). Post-Reformation Reformed Dogmatics (4 Vols). Grand Rapids: Baker Academic.

-Plantinga, A. (1998). Advise to Christian Philosophers. En J. Sennett (Ed.), The Analytic Theist. An Alvin Plantinga Reader. Grand Rapids: Eerdmans.

-Smith, J. (2009). Desiring the Kingdom. Grand Rapids: Baker Academic.

-Strauss, L. (2007). Cómo iniciarse en el estudio de la filosofía medieval. En L. Strauss, El renacimiento del racionalismo politico clásico. Buenos Aires: Amorrortu.

-Sudduth, M. (1995). The Prospects for "Mediate" Natural Theology in John Calvin. Religious Studies (31) 53-67.

-Trueman, C. \& Clark, R. S. (Eds.). (1999). Protestant Scholasticism. Essays in Reassesment. Carlisle: Paternoster.

-van Asselt, W. (Ed). (2010). Reformed Thought on Freedom. The Concept of Free Choice in Early Modern Reformed Theology. Grand Rapids: Baker Books.

-van Asselt, W. (2011). Introduction to Reformed Scholasticism. Grand Rapids: Reformation Heritage Books.

Sumario: Introducción; 1. Conocimiento de sí y de Dios en la Institución; 2. El acceso a Dios: sensus divinitatis y conocimiento desde la creación; 3. El conocimiento alcanzado; Conclusiones; Referencias. 